\title{
Green Service Level Agreement Compliance for Optical WDM Networks
}

\author{
Yashar Fazili $^{a *}$, Bill Robertson $^{b}$, William Phillips $^{\mathrm{c}}$ \\ a, band c Dalhousie University, Halifax, NS, Canada, B3H $4 R 2$
}

\begin{abstract}
In this paper we consider Green Service Level Agreement (SLA) as a constraint in finding a route for a dynamically received connection request in Wavelength Division Multiplexing (WDM) networks. We show that it is possible to save energy and reduce Greenhouse Gas (GHG) emission while satisfying optical network SLAs. The Integer Linear Programming (ILP) method introduced in this paper has been engineered to be simple and be solved in a timely manner needed for serving a dynamic connection request in control plane of the optical network. The ILP method introduced in this paper for the control plane of Optical WDM networks could also be used for the control plane of Software Defined Networks (SDN) and Software Defined Wide Area Networks.
\end{abstract}

Keywords: GMPLS, Green Networks, Control Plane;

\section{Introduction}

Per report in [1] $\mathrm{Co}_{2}$ Content of the atmosphere is increasing rapidly. Increasing $\mathrm{Co}_{2}$ content of the air is one of the most important reasons for global warming. IT industry among other industries has also stepped towards greener mechanisms to decrease the energy used and emission generated as a result of operating the networks and datacenters. Per report in [2] the Internet used 4 to 6 percent of the global energy produced in 2012, and therefore reducing the energy in IT and networking sector can be effective in reducing the $\mathrm{Co}_{2}$ emitted to the atmosphere. Green routing and the energy-efficient resource allocation mechanisms serve the purpose of reducing the energy and emission for optical networks that happen to be the backbone of the Internet. The Green Service Level Agreement introduced in $[3,4]$ have proposed a way to quantize and manage the reduction in energy usage and emission production that are requested by local and federal governments. Governments enforce the emission reduction as a result of their commitments to reduce the emission, after attending international summits on global warming as referenced in $[5,6]$. In this paper, we introduce a routing mechanism that considers Availability Service Level Agreement (ASLA) and Delay SLA (DSLA) needed to operate the optical network, and also considers a new Green SLA as detailed and explained in a later section. In a nutshell, we developed a simple ILP method that could be solved very quickly) so the control plane of the optical network does not become a bottleneck for serving the dynamic connection requests. Our model returns a result (A SLA compliant route or determining that there is no route when the current model is infeasible) in less than a second. This method can also be used in the control plane of a

* Corresponding author. Tel.: +9022232144

E-mail: yashar.fazili@dal.ca

(C) 2019 International Association for Sharing Knowledge and Sustainability.

DOI: $10.5383 / J U S P N .11 .02 .002$
Software Defined Network. The related work in this field comes next.

\section{Related Work}

The authors in $[7,8]$ have introduced a routing mechanism that simply "finds" a route that is most Energy Efficient (EE). The routing method of these papers finds a route that uses the least energy to connect the source node to the destination node. This task is accomplished by using the type of energy (green or nongreen) as a Traffic Engineering (TE) link characteristic. The authors of the paper in [9] introduce an Opaque Link-State Advertisement (LSA) that generates an "Energy Source Topology Database" used for calculating and finding the Energy Efficient route. This method does not consider any SLA, is abbreviated as EE, and has been reconstructed with the same name in this paper for performance comparison against our routing mechanisms. The authors in $[10,11]$ introduce a routing mechanism that computes a hybrid cost for a set of $\mathrm{k}$ routes and serves the connection request with a route that has the minimum of the hybrid cost. This method introduced as Emission Aware SLA Based (EASB) routing mechanism has been reconstructed for performance comparison in this paper. EASB considers the (ASLA) for calculating the route for a connection request. The paper in [12] introduces a Constrained Least Emission First (CLE) Routing mechanism that considers the ASLA and DSLA. This routing mechanism is the foundation of our new work and has a good reduction in the emission of the network. The paper in [13] introduces a new Opaque LSA that disseminates the greenness percentage of energy for each section of the network. The energy in each section of the network is a mix of the green and non-green source of energy. Therefore, considering a mix of energy is more accurate (and practical) than assuming the pure green or 
non-green source of energy in contrast to the assumption of authors in [7,9,14-16]. We use the same LSA of paper in [13] to build an energy and emission topology database for route computation and actual calculation of the emission. In this paper we use, $\rho$, as Energy Dirtiness. The higher the $\rho$ the more emission for the energy powering up an element of the network. For the simulation section of this paper, we used the same specification for optical elements of the forwarding layer explained in [17]. The list of the specification used is detailed in Table 1.

\begin{tabular}{|l|c|}
\hline \multicolumn{2}{|c|}{ Table 1. The energy needed for optical forwarding elements } \\
\hline \multicolumn{1}{|c|}{ Operation } & Power \\
\hline Inline Amplifier & $\begin{array}{l}50 \mathrm{~W} \text { for all } 96 \\
\text { Lambdas }\end{array}$ \\
\hline Signal Leveling Amplifier & $100 \mathrm{~W}$ \\
\hline $\begin{array}{l}\text { Switching each Lambda with Lambda } \\
\text { conversion using two transponders (OEO } \\
\text { operation) }\end{array}$ & $170 \mathrm{~W}$ \\
\hline $\begin{array}{l}\text { Adding or dropping each Lambda at the } \\
\text { source and the destination node }\end{array}$ & $85 \mathrm{~W}$ \\
\hline
\end{tabular}

No sleeping power state is considered in this paper (as it reduces the lifetime of the equipment) which is in contrasts with assumptions of authors of papers in [18-21]. The links and nodes that are not forwarding the lightpaths are turned OFF. In this paper, we are optimizing the energy usage for a forwarding layer of the optical network. Per information given in the papers of $[14,22]$ the energy consumed in electronic layer (control plane) of each core nodes is increased by about $3 \%$ only when a node is at $100 \%$ utilization level compared to idle condition and therefore can be considered as constant. The paper in [12] shows the energy model for each route and each link of the network which is used in this paper and is not repeated here.

\section{Green SLA-Aware Constrained Shortest Path}

\section{routing mechanism}

In this section, we introduce our proposed method. This method is the enhanced version of the routing method of our previous work in [12] called Constrained Least Emission (CLE) method. In this paper, The Enhance method is therefore called Enhanced Constrained Least Emission or ECLE. We would like to minimize the emission of the route which is a graph of $\mathrm{G}(\mathrm{A}, \mathrm{V})$ as illustrated in Equation (1). $\mathrm{M}_{\mathrm{ij}}$ is the emission value of each link as explained in [12]

$$
\sum_{i} \sum_{j} H_{i j} \cdot M_{i j} \cdot x_{i j} \quad \forall(i, j) \in A
$$

Subject to:

$\sum_{i} \sum_{j}\left(\ln A_{i j} \cdot x_{i j}\right) \geq-\ln (A S L A)$

$\sum_{i} \sum_{j} T_{i j} \cdot x_{i j} \leq D S L A$

$\left(1-\rho_{i j}\right) \cdot x_{i j} \leq G S L A$
All classical flow conservation shortest path problem constraints

$H_{i j}= \begin{cases}1 & \text { if link is } \text { ON } \\ 100 & \text { if link is } O F F\end{cases}$

$x_{i j}= \begin{cases}1 & \text { if links is used } \\ 0 & \text { if link is not used }\end{cases}$

Equations (2) - (4) are a set of constraints to ensure the ASLA, DSLA and GSLA respectively. $\boldsymbol{\rho}$ is the energy dirtiness of a link between node $\mathrm{i}$ and $\mathrm{j}$. Equation (5) gathers all flow conservation equations needed for constrained shortest path operation. Equation (6) shows the value of constant $\mathrm{H}_{\mathrm{ij}}$ to be multiplied by the original emission of a link $\left(\mathrm{M}_{\mathrm{ij}}\right)$. With this constant, the cost of the turned OFF links are 100 times more that ON links to ensure that ON links will be used first for calculating the route. Other links can be turned ON only if current ON link resources are exhausted and no route (that complies with the SLAs) can be computed using the turnedON links. If a route uses a link that is currently turned-OFF, the link is turned-ON when assigning the resources. Equation (7) sets the type of variable $\mathrm{x}_{\mathrm{ij}}$ to be binary. Next section details the setup of our simulation and defines our performance metrics used for comparing the routing mechanisms.

\section{Analysis}

\subsection{The Network}

The network used in this paper is the NSFnet with 14 nodes and 21 bidirectional links in authors of $[3,23,24]$ as shown in Fig. 1 from the paper in [25]. Connection request arrival is simulated using a Poisson process with an arrival rate of 20 connections per hour and the mean duration of 6 hours. The ASLA of each connection requests is between 0.999 to 0.99999. Per information in [26], the total delay of the North American network of NTT Data is less than $50 \mathrm{~ms}$. We divided the $50 \mathrm{~ms}$ over 4 time zones show in Fig. 1 to obtain $12.5 \mathrm{~ms}$ delay per each zone and use it as the DSLA of the connection request. Therefore, a lightpath passing through two time zones may have a total of $25 \mathrm{~ms}$ delay. In this paper, connection requests may ask for a minimum of $30 \%$ and up to $40 \%$ greenness of the links. Each link has 96 Lambdas with one Lambda per each lightpath per hop. When the last Lambda (resource) of a link is assigned, it is taken out from the dynamic network and it is not considered for any new routing. By taking out the exhausted link, ILP method can work with one less constraint which is finding a route through links that have available resources. The ILP is solved by Gurobi optimizer in [27].

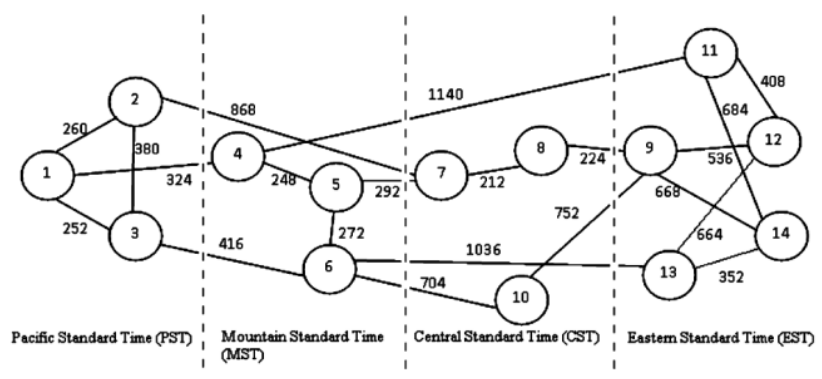




\section{Fig. 1 NSFnet network}

\subsection{Performance Metrics}

In this section, we introduce the performance metrics that we used for benchmarking the newly proposed routing mechanism.

\section{Success Rate}

The ratio of the total number of served connections requests over the total number of connection requests. A higher number is desired for this metric.

\section{ASLA Satisfaction (ASLS)}

The ratio of the total number of served connections requests that received a route that met their ASLA over the total number of served connection requests. A higher number is desired for this metric as indicates higher ASLA compliance.

\section{DSLA Satisfaction (DSLS)}

The ratio of the total number of served connections requests that received a route that met their DSLA over the total number of served connection requests. A higher number is desired for this metric as indicates higher DSLA compliance. The higher the value the higher the DSLA compliance.

\section{GSLA Satisfaction (GSLS)}

The ratio of the total number of served connections requests that were served with a route that met their GSLA over the total number of the served connection requests. A higher number is desired for this metric as it indicates higher GSLA compliance. The Routing mechanism introduced in this paper considers the GSLA in finding a route, therefore, is expected to provide $100 \%$ GSLA satisfaction

\section{Success Satisfaction}

This metric is the product of Success Rate, ASLS, DSLS and GSLS and gives an overall satisfaction rate of all served connections. The fact that a routing mechanism gives $100 \%$ success does not mean a higher or $100 \%$ Success Satisfaction. The lower Success Satisfaction means it would be better to reject the connection request as opposed to establishing the connection with a route that does not comply with SLAs of the connection request.

\section{Node Power}

The total power draw by the forwarding plane of all nodes of the network. Continuity constraint in assigning the resources or Lambdas of the route can lower the power consumption in the nodes. The proposed method of this paper uses the continuity constraint is assigning the resources. The number in this graph is in units of $\mathrm{kW}$.

\section{Link Power}

The total power draw by links of the network. With the proposed method of this paper, ON links are used first leaving more links in OFF state, resulting in a lower energy usage in links. The number in this graph is in units of $\mathrm{kW}$.

\subsection{Routing Methods Used in Benchmarking}

We have compared our proposed ECLE method against EE and EASB. The non-green method of our paper in [12] which is called Multi-Constrained Shortest Path (MCSP) has also been used for analysis. MCSP does not consider any energy- related information (LSAs) and finds the shortest path for a connection request with ASLA and DSLA Satisfaction constraints to be met.

\subsection{Results}

In this section, we present the results of our simulation and corresponding analysis. Fig. 2 shows the results for the success rate. As we can see in this figure, EE with no constraint in finding a route provides $100 \%$ success rate which is expected. Adding the constraint of ASLA to EASB and ASLA and DSLA to MCSP drop the success rate by about $25 \%$ as route needs to conform to more constraints. Ultimately adding the GSLA further drops the success rate by $20 \%$ giving the total of 55\% success rate to ECLE. The 55\% success rate and \%75 percent success rate may seem to be low but as we will see in later figures for various SLA satisfactions, routing methods that consider the SLAs in finding a route give higher or $100 \%$ satisfaction for SLAs they recognize. For example, Fig. 3 show $100 \%$ ASLS for EASB, MCSP, and ECLE. Without considering ASLA, ASLS ratio can drop by more than $40 \%$ as we can see in Fig. 3 for EE. Fig. 4. Shows the results for DSLS. In this figure ECLE that considers the DSLA constraint and provisions the lightpath with continuity constraint, provides $100 \%$ Delay SLA satisfaction or DSLS ratio. MCSP without the continuity constraint for Lambda assignment of lightpath gives $95 \%$ percent satisfaction. Without considering the DSLA for route calculation, DSLS ratio can drop by more than 25\% when using EE and EASB. Although MCSP considers DSLA constraint for finding a route it does not give $100 \%$ satisfaction due to the fact that Lambda conversion delay in each node adds up to the total delay of the lightpath. This argument has been further explained in the paper of [12]. Fig. 5 shows the results of the analysis for GSLS. As we can see in this figure ECLE provides $100 \%$ GSLA satisfaction as it considers the GSLA constraint in finding a route. Without GSLA constraint in finding a route, GSLS ratio can drop by about $30 \%$ for EASB and MCSP and around $25 \%$ by EE. The interesting fact about this graph is. EE* cannot give $100 \%$ satisfaction for GSLA. This observation as also emphasized in the paper of [3] shows that more investment in green energy is needed. Fig. 6 shows the results for Success Satisfaction which is the overall satisfaction of the served routes. As we can see in this figure although ECLE has about 55\% success rate but since it provides $100 \%$ SLA satisfaction for all SLAs, it gives about $4 \%$ better overall satisfaction compared to MCSP that has about $15 \%$ higher success rate. ECLE gives more than $15 \%$ better overall satisfaction compared to EASB. Fig. 6 ultimately suggests that there is no point of establishing the connection and provision the resources when the route does not comply with SLAs. Fig. 7 shows the total number of active or turned ON links of the network. AS we stated before, ECLE tries to mostly use the ON links. Therefore, this behavior makes the ECLE to use the least number of the active edges as we can see in Fig. 7. With this behavior ECLE forms a "Highway" across the ON links, turning ON the OFF links only when resources of ON links are exhausted or no route that complies with SLAs could be computed using ON links. Less number of turned ON links means lower energy usage in links of the network as we can see in Fig. 8, ECLE draws about $4 \mathrm{~kW}$ less energy compared to EE and close to $7 \mathrm{~kW}$ less energy compared to non-green MCSP. ECLE establishes the lightpaths with continuity constraint, therefore, no Lambda conversion is performed in the intermediate nodes of the lightpaths. This fact

\footnotetext{
* Which purely and without any constraint choses the most Energy Efficient route for a connection request
} 
results in reduced energy usage in nodes of the network as we can see in Fig. 9 and Fig. 10. Fig. 9 shows zero number of Lambda conversion for ECLE which is translated into $10 \mathrm{~kW}$ power usage, which is $67 \%$ less than MCSP with around 30 $\mathrm{kW}$ power usage in nodes. In other words, although MCSP has $20 \%$ better success rate but is uses three times the energy to establish the lightpaths. Finally, lower energy usage in nodes and links of the network results in lower average energy per unit resource Lambda as shown in Fig. 11. ECLE consumes about 33\% less energy compared to the non-green MCSP and close to $31 \%$ less energy compared to EASB. At the end, we can conclude that considering the SLAs in finding a route may reduce the success rate of the network, however, can result in a better overall success satisfaction. Our ECLE provided 100\% SLA satisfaction for all SLAs of this paper and used up to $33 \%$ less energy compared to the non-green method.

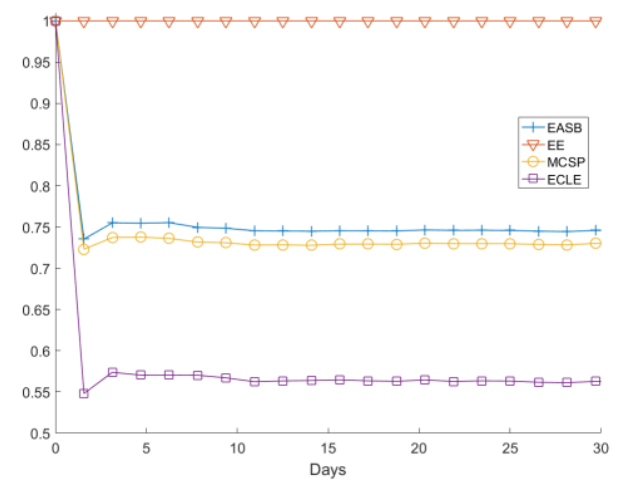

Fig. 2 Success Rate

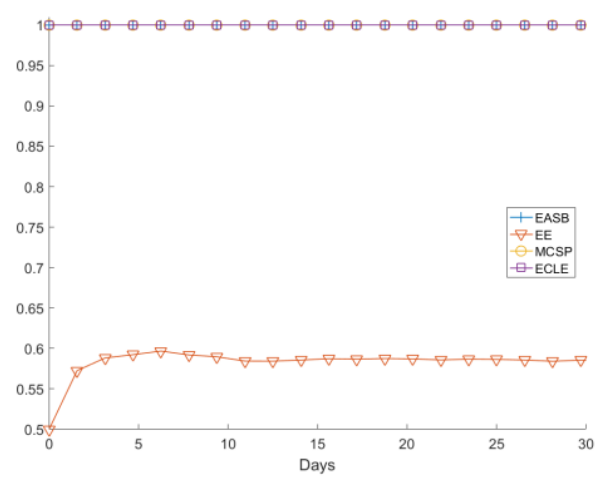

Fig. 3 ASLS ratio

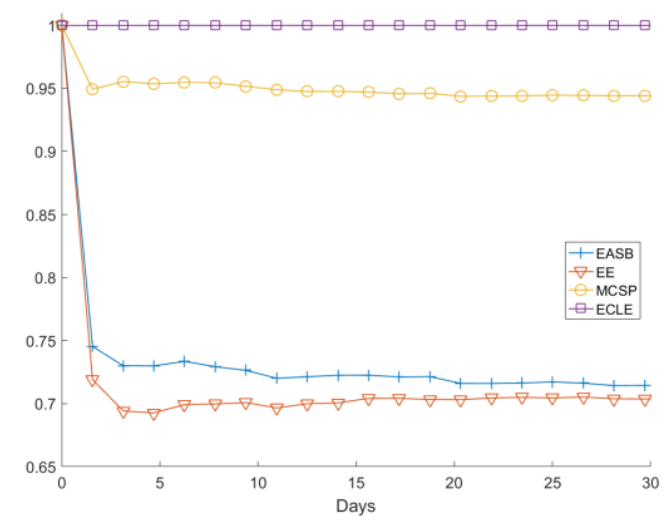

Fig. 4 DSLS ratio

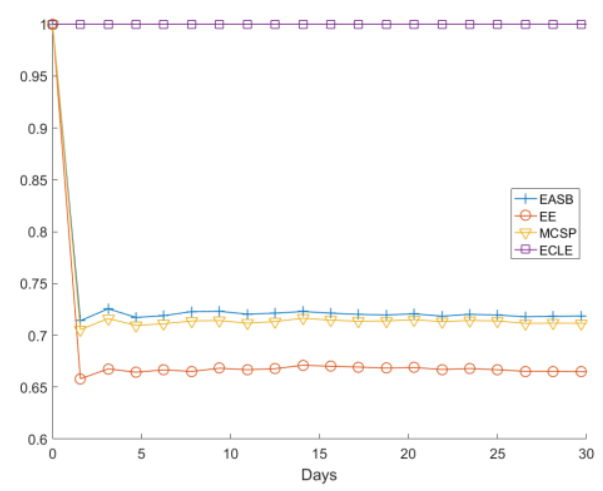

Fig. 5 GSLS ratio

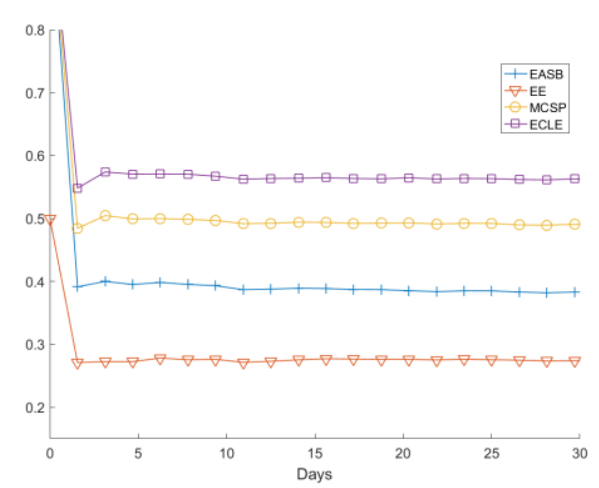

Fig. 6 Success satisfaction ratio

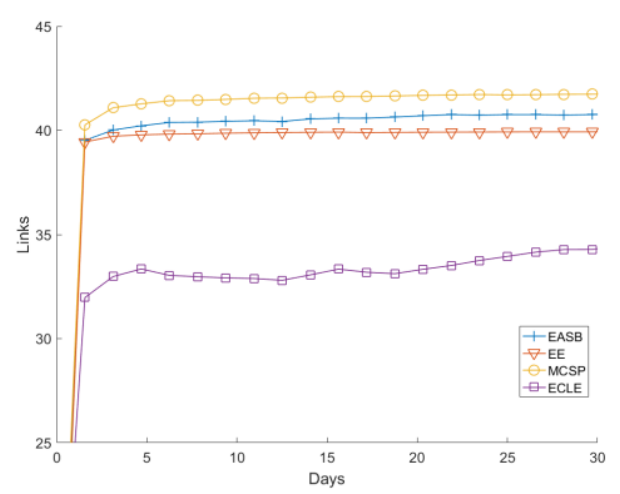

Fig. 7 Number of active edges

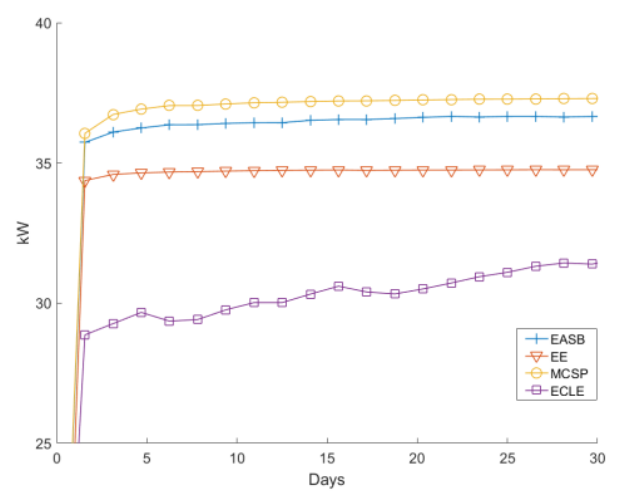


Fig. 8 Link power

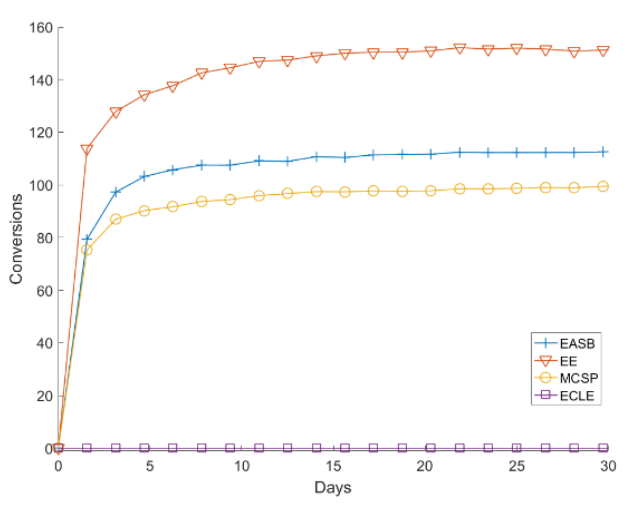

Fig. 9 Number of Conversions

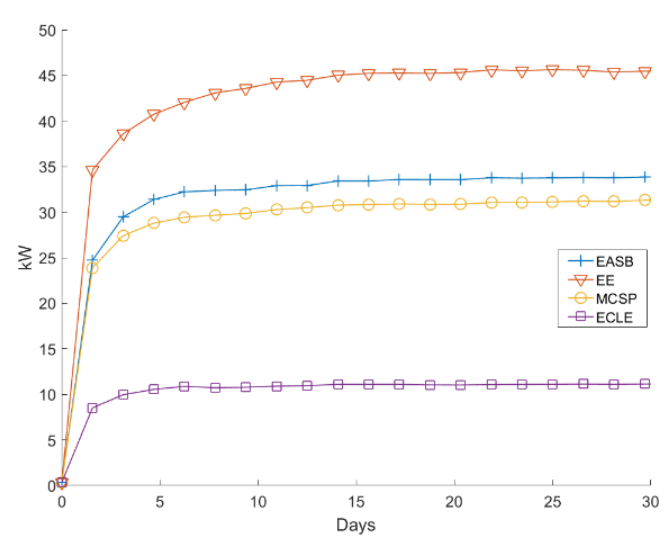

Fig. 10 Node power

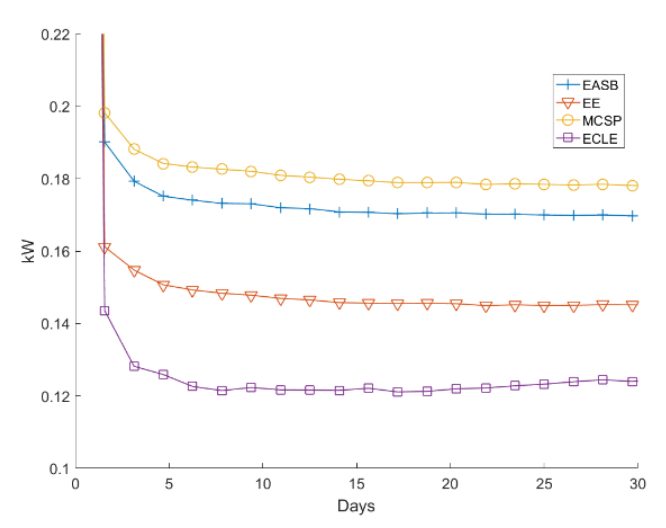

Fig. 11 kW per Lambda

\section{Conclusion}

In this paper, we introduced an ILP method that considers ASLA, DSLA and GSLA that can resolve to a decision (a route or no feasibility) in less than a second as needed for dynamic and real-time connection requests. Using the introduced method of this paper, Service Provides can provision resources for lightpaths with up to $33 \%$ less energy usage for better success satisfaction. Using the method of this paper Service provides can save the resource (Lambdas) and not to provision and establish the lightpath when the lightpath is not in compliance with all required SLAs. Although links are turned $\mathrm{ON}$ by nodes of the network and have the same energy greenness as the links they power, we will include their greenness for route calculation in future work to obtain even more accurate results for energy consumption. We will compare the performance if ECLE with other routing mechanisms in terms of Quality of Service (QoS) for data and media streaming applications as detailed in [28]. In future, we are going to introduce the service types as detailed in the paper of [29] as constraints for aggregate lightpaths and performed the same analysis. This allows the green routing method to also be Service Aware.

\section{References}

[1] Blasing TJ. Recent Greenhouse Gas Concentrations. Recent Greenhouse Gas Concentrations 2015, U.S. Department of Energy.2015, From http://cdiac.ornl.gov/pns/current_ghg.html; DOI: $10.3334 / C D I A C / a t g .032$ https://doi.org/10.3334/CDIAC/atg.032

[2] Strickland J. How much energy does the Internet use?. 2012; Available at:

http://computer.howstuffworks.com/internet/basics/how-muchenergy-does-internet-use2.htm. Accessed September, 8, 2016.

[3] Fazili Y, Nafarieh A, Raza MH, Robertson B, Philips W. The effect of adopting green SLA on key parameters of optical WDM networks. Journal of Ambient Intelligence and Humanized Computing 2016;7(3):371-384. https://doi.org/10.1007/s12652-016-0364-5

[4] Laszewski GV, Wang L. Green IT Service Level Agreements. Grids and Service-Oriented Architectures for Service Level Agreements: Springer US; 2010. p. 77. https://doi.org/10.1007/978-1-4419-7320-7_8

[5] CBC. U.S., China agree to cut greenhouse gases in bid to spur others to join. 2014; Available at: http://www.cbc.ca/news/politics/u-s-china-agree-to-cutgreenhouse-gases-in-bid-to-spur-others-to-join-1.2832053; Accessed Feb 3, 2015.

[6] Mooney SM C. In a major moment for climate policy, China, Brazil, and the U.S. all announce new commitments. 2015; Available at: https://www.washingtonpost.com/news/energyenvironment/wp/2015/06/30/china-brazil-and-the-u-s-allannounce-new-climate-and-clean-energy-goals/;. Accessed Jun 30, 2015.

[7] Wang J. et al; Energy-aware routing optimization in dynamic GMPLS controlled optical networks. Transparent Optical Networks (ICTON), 2012 14th International Conference on; 2012.

https://doi.org/10.1109/ICTON.2012.6253843

[8] Wang J. et al; Green-aware routing in GMPLS networks. Computing, Networking and Communications (ICNC), 2012 International Conference on; 2012. https://doi.org/10.1109/ICCNC.2012.6167416

[9] Wang J. et al; OSPF-TE extensions for green routing in optical networks. Opto-Electronics and Communications 
Conference (OECC), 2012 17th; 2012. https://doi.org/10.1109/OECC.2012.6276497

[10] Fazili Y, Nafarieh A, Robertson W. Table Driven Hybrid Energy-aware and SLA-based Routing Mechanism over Optical Networks. Procedia Computer Science; The 5th International Conference on Ambient Systems, Networks and Technologies (ANT-2014) 2014;32:1037-1043. https://doi.org/10.1016/j.procs.2014.05.530

[11] Fazili Y, Nafarieh A, Robertson W. Hybrid Energy-aware and SLA-based Routing Mechanism over Optical Networks. Procedia Computer Science; The 4th International Conference on Ambient Systems, Networks and Technologies (ANT 2013) 2013;19:1151-1158. https://doi.org/10.1016/j.procs.2013.06.164

[12] Fazili Y, Nafarieh A, Robertson B. Multi SLA Aware Green Provisioning Mechanism for $\backslash W D M \backslash$ Networks. Procedia Computer Science 2016;94:129. https://doi.org/10.1016/j.procs.2016.08.021

[13] Nafarieh A, Fazili Y, Raza M, Robertson W. Greenness Link State Advertisement Extension for $\backslash W D M \backslash$ Networks.

Procedia Computer Science 2016;94:310.

https://doi.org/10.1016/j.procs.2016.08.046

[14] Ricciardi S, et al. Energy-Aware RWA for WDM Networks with Dual Power Sources. 2011 IEEE International Conference on Communications (ICC); 2011.

https://doi.org/10.1109/icc.2011.5962432

[15] Ricciardi S, Wang J, Palmieri F, Careglio D, Dittman L. An energy-aware engineered control plane for wavelengthrouted networks. Transactions on Emerging

Telecommunications Technologies 2015;26(2):231-249. https://doi.org/10.1002/ett.2815

[16] Amaldi E, Capone A, Gianoli LG. Energy-aware UIP traffic engineering with shortest path routing. Computer Networks 2013;57(6):1503.

https://doi.org/10.1016/j.comnet.2013.02.006

[17] ADVA. Scalable Optical Transport. 2016; Available at http://www.advaoptical.com/en/products/scalable-opticaltransport.aspx. Accessed Apr, 10, 2016.

[18] Musumeci F, Tornatore M, Lopez Vizcaino J, Ye Y, Pattavina A. Energy-efficiency of Protected IP-over-WDM Networks with Sleep-mode Devices. J.High Speed Netw. 2013 jan;19(1):19-32.

[19] F. Musumeci, M. Tornatore, A. Pattavina. A Power Consumption Analysis for IP-Over-WDM Core Network Architectures. IEEE/OSA Journal of Optical Communications and Networking 2012;4(2):108-117.

https://doi.org/10.1364/JOCN.4.000108

[20] Muhammad A. Energy-Efficient WDM Network Planning with Dedicated Protection Resources in Sleep Mode. Global Telecommunications Conference (GLOBECOM 2010), 2010 IEEE; 2010.

https://doi.org/10.1109/GLOCOM.2010.5683205

[21] Cianfrani A, et al. An OSPF enhancement for energy saving in IP networks. Computer Communications Workshops
(INFOCOM WKSHPS), 2011 IEEE Conference on; 2011. https://doi.org/10.1109/INFCOMW.2011.5928832

[22] Aleksić S. Analysis of Power Consumption in Future High-Capacity Network Nodes. IEEE/OSA Journal of Optical Communications and Networking 2009;1(3):245-258. https://doi.org/10.1364/JOCN.1.000245

[23] Song Y. et al. Energy-aware path selection for scheduled lightpaths in IP-over-WDM networks. 2011 18th IEEE Symposium on Communications and Vehicular Technology in the Benelux (SCVT); 2011.

[24] Charbonneau N, Vokkarane VM. Performance modeling of HS-RR-TCP over load-balanced optical burst-switched (OBS) networks. Optical Switching and Networking 2011 4;8(2):116-128. https://doi.org/10.1016/j.osn.2010.10.002

[25] X. Dong, T. El-Gorashi, J. M. H. Elmirghani. IP Over WDM Networks Employing Renewable Energy Sources. Journal of Lightwave Technology 2011;29(1):3-14. https://doi.org/10.1109/JLT.2010.2086434

[26] NTT. Global IP Network SLA. 2016; Available at: http://www.us.ntt.net/support/sla/network.cfm. Accessed Nov, $5,2015$.

[27] Gurobi. Gurobi Optimizer 6.5. 2017; Available at: http://www.gurobi.com/.

[28] Al Mamun A., Sheltami T.R, Ali H., Anwar S. Performance Evaluation of Routing Protocols for Video Conference over MPLS VPN Network. Journal of Ubiquitous Systems \& Pervasive Networks, Volume 7, No. 1 (2016) pp. 01-06 DOI: 10.5383/JUSPN.07.01.001

[29] Abdelaziz J., Addab A., Mcheick H. An architecture model for fog computing. Journal of Ubiquitous Systems \& Pervasive Networks, Volume 10, No. 1 (2018) pp. 21-25, DOI: 10.5383/JUSPN.10.01.003 https://doi.org/10.5383/JUSPN.10.01.003 